\title{
Variational Calculation of the Doubly-Excited States Nsnp of He-Like Ions via the Modified Atomic Orbitals Theory
}

\author{
Abdourahmane Diallo1*, Ibrahima Sakho², Jean Kouhissoré Badiane1, \\ Mamadou Diouldé Ba1 ${ }^{1}$, Modou Tine ${ }^{1}$ \\ ${ }^{1}$ Department of Physics, UFR Sciences and Technologies, University Assane Seck of Ziguinchor, Ziguinchor, Senegal \\ ${ }^{2}$ Department of Physics and Chemistry, UFR Sciences and Technologies, University of Thiès, Thiès, Senegal \\ Email: *a.diallo1483@zig.univ.sn
}

How to cite this paper: Diallo, A., Sakho, I., Badiane, J.K., Ba, M.D. and Tine, M. (2021) Variational Calculation of the Doubly-Excited States Nsnp of He-Like Ions via the Modified Atomic Orbitals Theory. Journal of Modern Physics, 12, 105-121.

https://doi.org/10.4236/jmp.2021.122011

Received: August 6, 2020

Accepted: January 24, 2021

Published: January 27, 2021

Copyright $\odot 2021$ by author(s) and Scientific Research Publishing Inc. This work is licensed under the Creative Commons Attribution International License (CC BY 4.0).

http://creativecommons.org/licenses/by/4.0/

\begin{abstract}
In this paper, we have declined the formalism of the method of the Modified Atomic Orbital Theory (MAOT) applied to the calculations of energies of doubly excited states $2 s n p, 3 s n p$, and $4 \operatorname{snp}$ Helium-like systems. Then we also applied the variational procedure of the Modified Atomic Orbital Theory to the computations of total energies, excitation energies of doubly-excited states $2 \operatorname{snp}, 3 \operatorname{snp}, 4 \operatorname{snp}$ types of Helium-like systems. The results obtained in this work are in good agreement with the experimental and theoretical values available.
\end{abstract}

\section{Keywords}

Modified Atomic Orbital Theory, Doubly Excited States, Excitation Energy, Helium-Like Systems

\section{Introduction}

Much theoretical research has revealed that the helium atom exhibits a strong electron-electron correlation. Since the early experiment by Madden and Codling [1], Madden and Ederer [2], and theoretical explanation by Cooper et al. [3], doubly-excited states of helium-like atoms have been the target of a number of theoretical approaches. The increasing interest of physicists in these studies over the years is connected with the understanding of collisional and radiational processes which take place in hot astrophysical and laboratory plasma [4]. The greatest attention has been concentrated on the study of doubly-excited states [5]. Some of these doubly-excited states in two-electron systems have been iden- 
tified in the solar flare [6] and in the solar corona [7] and revealed experimentally by the studies of double Rydberg resonances in negative ions of rare gases [8] [9]. The investigations of the intrashell S states of two-electron systems are advanced and due to the group theoretical method [10] [11] which allowed intrashell states to be approximately classified and some of these properties were studied [12] [13].

So, most atomic spectra can be treated in term of singly excitation of singly or mixed configurations [14] [15]. After Herrick and Sinanoglu [11], higher-energy Rydberg envelopes contain doubly-excited states which are generally labelled in the usual spectroscopic notation $\left(N l, n l^{\prime}\right)^{2 S+1} L^{\pi}$ with $n=N, N+1, \cdots$. In these notations, $N$ and $n$ denote respectively the principal quantum numbers of the inner and the outer electron, $I$ and $P$ are respectively orbital quantum numbers, $S$ the total spin, $L$ the total angular momentum and $\pi$ the parity of the system.

Various methods have been performed to understand electron-electron correlation effects in doubly $\left(N l, n l^{\prime}\right)^{2 S+1} L^{\pi}$ excited states of He-like systems. Although many accurate data have been tabulated for these doubly excited states, the methods used require in general, complexity in the Variationnal procedure along with the use of computational codes.

Many theoretical studies have been done on doubly-excited states $\left(N l, n l^{\prime}\right)^{2 S+1} L^{\pi}$. Among these methods, we have the theoretical and experimental methods [16]-[23]. The variational method of time-independent perturbation from Ray and Mukherjee was applied for the calculation of the total energies of the $2 \mathrm{~s}^{2}, 2 \mathrm{p}^{2}$ and $3 \mathrm{~d}^{2}$ states of $\mathrm{He}, \mathrm{Li}^{+}, \mathrm{Be}^{2+}$, and $\mathrm{B}^{3+}$ [24]. Sakho used the semi-empirical procedure of the Screening Constant by Unit Nuclear Chargemethod (SCUNC) to calculate the energies of doubly excited states (Nsnp) ${ }^{1} \mathrm{P}_{\mathrm{o}}$ helium-like systems [25].

In all these ab initio methods, energies of $\left(N l, n l^{\prime}\right)^{2 S+1} L^{\pi}$ doubly-excited states of $\mathrm{He}$ isoelectronic sequence can't be expressed in an analytical formula. In addition, most of these preceding methods require large basis-set calculations involving a fair amount of mathematics complexity.

The Modified Atomic Orbital Theory is a purely theoretical method initiated by Sakho [26]. This theory stems from Slater's orbital theory [27]. This theory (MAOT) has been known for its simplicity, as it is a very suitable calculation method that has yielded enormous results from simple semi-empirical formulas without resorting to a computer program in solving resonant photoionization problems. It was subsequently that Sakho [25] studied the resonance energies of the Rydberg series of $2 \mathrm{~s}^{2} 2 \mathrm{p}^{4}\left({ }^{1} \mathrm{D}_{2}\right) n \mathrm{~s}, n \mathrm{~d}, 2 \mathrm{~s}^{2} 2 \mathrm{p}^{4}\left({ }^{1} \mathrm{~S}_{0}\right) n \mathrm{~s}, n \mathrm{~d}$, and $2 \mathrm{~s}^{2} 2 \mathrm{p}^{5}\left({ }^{3} \mathrm{P}_{2}\right)$ states from of the metastable $2 \mathrm{~s}^{2} 2 \mathrm{p}^{5}\left({ }^{2} \mathrm{P}_{1 / 2}\right)$ state and the ground $2 \mathrm{~s}^{2} 2 \mathrm{p}^{5}\left({ }^{2} \mathrm{P}_{3 / 2}\right)$ state of the $\mathrm{Ne}^{+}$ion. Thus the variational principle, which is a purely theoretical method, takes advantage of the principle of variation. This variational method is a computational technique to provide approximate solutions to solving the Schrödinger equation. In the following, after a brief review of Slater's orbital theory, we apply for the first time the variational procedure of the Modified 
Atomic Orbitals Theory to the calculations of total energies, excitation energies of doubly excited $2 \mathrm{~s} n \mathrm{p}, 3 \mathrm{~s} n \mathrm{p}$, $4 \mathrm{~s} n \mathrm{p}$ states of types helium-like systems. This procedure consists of determining the variational parameter $\alpha$ and the screening constant $\sigma$, from the construction of a correlated wave function.

\section{Theory}

\subsection{Brief Description of the Modified Atomic Orbitals Theory}

In the context of the Modified Atomic Orbitals Theory (MAOT), the total energy of a $(v \ell)$-given orbital is expressed as Rydberg units [28].

$$
E(v \ell)=-\frac{[Z-\sigma(\ell)]^{2}}{v^{2}} .
$$

For the $\left(N l, n l^{\prime}\right)^{2 S+1} L^{\pi}$ doubly excited states, the total energy of an atomic system of many $\mathrm{M}$ electrons is expressed as follows

$$
E=-\sum_{i=1}^{M} \frac{\left[Z-\sigma_{i}\left({ }^{2 S+1} L^{\pi}\right)\right]^{2}}{v_{i}^{2}} .
$$

\subsection{Construction of the Wave Function}

In the construction of the correlated wave function, a product of hydrogen-type wave functions is performed in which variational parameters are introduced. Thus, in the case of atomic systems, these criteria are generally determined by the screen effects exerted by the electrons on each other by the spin-orbit interaction, etc.

The hydrogen wave functions for $\left|n, l, m_{l}\right\rangle$ states are radial and have the same shape. They are non-normed and it's obtained from the radial coordinates $(r)$ and an exponential factor.

So for different states, we get:

For $4 \mathrm{~s}(1=0)$ :

$$
R_{4,0}(r)=\frac{24}{96} \times\left(\frac{Z}{a_{0}}\right)^{\frac{3}{2}} \times\left(1-\frac{3 \times Z}{4 \times a_{0}} \times r+\frac{Z^{2}}{8 \times a_{0}^{2}} \times r^{2}-\frac{Z^{3}}{192 \times a_{0}^{3}} \times r^{3}\right) \times \mathrm{e}^{-\frac{Z \times r}{4 \times a_{0}}}
$$

For $4 \mathrm{p}(l=1)$ :

$$
R_{4,1}(r)=\frac{5}{16 \sqrt{2}} \times\left(\frac{Z}{a_{0}}\right)^{\frac{3}{2}} \times\left(\frac{Z}{a_{0}} \times r-\frac{1}{4} \times \frac{Z^{2}}{a_{0}^{2}} \times r^{2}+\frac{1}{80} \times \frac{Z^{3}}{a_{0}^{3}} \times r^{3}\right) \times \mathrm{e}^{-\frac{Z \times r}{4 \times a_{0}}}
$$

For $3 s(1=0)$ :

$$
R_{3,0}(r)=\frac{1}{3 \sqrt{3 \pi}} \times\left(\frac{z}{a_{0}}\right)^{\frac{3}{2}} \times\left(1-\frac{2}{3} \times \frac{Z}{a_{0}} \times r+\frac{2}{27} \times \frac{Z^{2}}{a_{0}^{2}} \times r^{2}\right) \times \mathrm{e}^{-\left(\frac{Z \times r}{3 \times a_{0}}\right)}
$$

For $3 p(l=1)$ :

$$
R_{3,1}(r)=\frac{2 \times \sqrt{2}}{27 \times \sqrt{\pi}} \times\left(\frac{Z}{a_{0}}\right)^{\frac{3}{2}} \times\left(\frac{Z}{a_{0}} \times r-\frac{Z^{2}}{6 \times a_{0}^{2}} \times r^{2}\right) \times \mathrm{e}^{-\left(\frac{Z \times r}{3 \times a_{0}}\right)}
$$


For $2 \mathrm{~s}(I=0)$ :

$$
R_{2,0}(r)=\frac{2}{4 \times \sqrt{2 \pi}} \times\left(\frac{Z}{a_{0}}\right)^{\frac{3}{2}} \times\left(1-\frac{Z}{2 \times a_{0}} \times r\right) \times \mathrm{e}^{-\left(\frac{Z \times r}{2 \times a_{0}}\right)}
$$

For $2 \mathrm{p}(I=1)$

$$
R_{2,1}(r)=\frac{1}{4 \times \sqrt{2 \pi}} \times\left(\frac{Z}{a_{0}}\right)^{\frac{3}{2}} \times\left(\frac{Z}{a_{0}} \times r\right) \times \mathrm{e}^{-\left(\frac{Z \times r}{2 \times a_{0}}\right)}
$$

To build the wave functions of $\left(N l, n l^{\prime}\right)^{2 S+1} L^{\pi}$ type, the product of the radial portions $R_{n, l}(r)$ is produced while considering the electrons (1) and (2) heliumoid systems, whose radial coordinates are respectively $r_{1}$ and $r_{2}$. As part of the independent particle model where electronic correlation phenomena are neglected, (Coulombian repulsion, spin-orbit interaction, etc.), the product of the functions is given as follows:

For the function $2 \mathrm{~s} 2 \mathrm{p}$ :

$$
\begin{aligned}
& 2 \mathrm{~s}=\left(1-\frac{Z}{2 \times a_{0}} \times r_{1}\right) \times \mathrm{e}^{-\left(\frac{Z \times r_{1}}{2 \times a_{0}}\right)} \text { and } 2 \mathrm{p}=\left(\frac{Z}{a_{0}} \times r_{2}\right) \times \mathrm{e}^{-\left(\frac{Z \times r_{2}}{2 \times a_{0}}\right)} \\
& \Psi(2 \mathrm{~s} 2 \mathrm{p})=\left[\left(1-\frac{Z}{2 \times a_{0}} \times r_{1}\right) \times\left(\frac{Z}{a_{0}} \times r_{2}\right)\right] \times \mathrm{e}^{-\frac{Z}{2 \times a_{0}} \times r_{1}} \times \mathrm{e}^{-\frac{Z}{2 \times a_{0}} \times r_{2}}
\end{aligned}
$$

For the function 3s3p:

$$
\begin{aligned}
& 3 \mathrm{~s}=\left(1-\frac{2 \times Z}{3 \times a_{0}} \times r_{1}+\frac{2 \times Z^{2}}{27 \times a_{0}^{2}} r_{1}^{2}\right) \times \mathrm{e}^{-\left(\frac{Z}{3 \times a_{0}} \times r_{1}\right)} \text { and } \\
& 3 \mathrm{p}=\left(\frac{Z}{a_{0}} \times r_{2}-\frac{Z^{2}}{6 \times a_{0}^{2}} \times r_{2}^{2}\right) \times \mathrm{e}^{-\left(\frac{Z}{3 \times a_{0}} \times r_{2}\right)} \\
& \Psi(3 \mathrm{~s} 3 \mathrm{p})=\left(\left(1-\frac{2 \times Z}{3 \times a_{0}} \times r_{1}+\frac{2 \times Z^{2}}{27 \times a_{0}^{2}} r_{1}^{2}\right) \times\left(\frac{Z}{a_{0}} \times r_{2}-\frac{Z^{2}}{6 \times a_{0}^{2}} \times r_{2}^{2}\right)\right) \\
& \times \mathrm{e}^{-\left(\frac{Z}{3 \times a_{0}} \times r_{1}\right)} \times \mathrm{e}^{-\left(\frac{Z}{3 \times a_{0}} \times r_{2}\right)}
\end{aligned}
$$

For the function 4s4p:

$$
\begin{aligned}
4 \mathrm{~s} & =\left(1-\frac{3 \times Z}{4 \times a_{0}} \times r+\frac{Z^{2}}{8 \times a_{0}^{2}} \times r^{2}-\frac{Z^{3}}{192 \times a_{0}^{3}} \times r^{3}\right) \times \mathrm{e}^{-\left(\frac{Z \times r_{1}}{4 \times a_{0}}\right)} \\
4 \mathrm{p} & =\left(\frac{Z}{a_{0}} \times r-\frac{1}{4} \times \frac{Z^{2}}{a_{0}^{2}} \times r^{2}+\frac{1}{80} \times \frac{Z^{3}}{a_{0}^{3}} \times r^{3}\right) \times \mathrm{e}^{-\left(\frac{Z \times r_{2}}{4 \times a_{0}}\right)} \\
\Psi(4 \mathrm{~s} 4 \mathrm{p}) & =\left(\left(1-\frac{3 \times Z}{4 \times a_{0}} \times r_{1}+\frac{Z^{2}}{8 \times a_{0}^{2}} \times r_{1}^{2}-\frac{Z^{3}}{192 \times a_{0}^{3}} \times r_{1}^{3}\right)\right. \\
& \left.\times\left(\frac{Z}{a_{0}} \times r_{2}-\frac{1}{4} \times \frac{Z^{2}}{a_{0}^{2}} \times r_{2}^{2}+\frac{1}{80} \times \frac{Z^{3}}{a_{0}^{3}} \times r_{2}^{3}\right)\right) \times \mathrm{e}^{-\left(\frac{Z \times r_{1}}{4 \times a_{0}}\right)} \times \mathrm{e}^{-\left(\frac{Z \times r_{2}}{4 \times a_{0}}\right)}
\end{aligned}
$$


Taking into account the phenomena of electron-electron correlation effects occurring in He-like systems, the nuclear charge of the exponential factor is substituted in favor of the effective charge $Z$, and in atomic unit, the Bohr radius $a_{0}=1$.

So these functions become:

For the wave function $2 \mathrm{~s} n \mathrm{p}$ :

$$
\Psi(2 \text { snp })=\left(\left(1-\frac{Z}{2 \times a_{0}} \times r_{1}\right) \times\left(\frac{Z}{a_{0}} \times r_{2}\right)\right) \times \mathrm{e}^{-\frac{Z^{*}}{n}\left(r_{1}+r_{2}\right)}
$$

For the wave function 3 s np:

$$
\Psi(3 \text { snp })=\left(\left(1-\frac{2 \times Z}{3 \times a_{0}} \times r_{1}+\frac{2 \times Z^{2}}{27 \times a_{o}^{2}} r_{1}^{2}\right) \times\left(\frac{Z}{a_{0}} \times r_{2}-\frac{Z^{2}}{6 \times a_{0}^{2}} \times r_{2}^{2}\right)\right) \times \mathrm{e}^{-\frac{Z^{*}}{n}\left(\eta_{1}+r_{2}\right)}
$$

For the wave function $4 \mathrm{~s} n \mathrm{p}$ :

$$
\begin{aligned}
\Psi(4 \mathrm{snp})= & \left(\left(1-\frac{3 \times Z}{4 \times a_{0}} \times r_{1}+\frac{Z^{2}}{8 \times a_{0}^{2}} \times r_{1}^{2}-\frac{Z^{3}}{192 \times a_{0}^{3}} \times r_{1}^{3}\right)\right. \\
& \left.\times\left(\frac{Z}{a_{0}} \times r_{2}-\frac{1}{4} \times \frac{Z^{2}}{a_{0}^{2}} \times r_{2}^{2}+\frac{1}{80} \times \frac{Z^{3}}{a_{0}^{3}} \times r_{2}^{3}\right)\right) \times \mathrm{e}^{-\frac{Z^{*}}{n}\left(r_{1}+r_{2}\right)}
\end{aligned}
$$

where the effective charge number $Z$ is given by:

$$
Z^{*}=Z\left(1-\frac{\sigma\left(N l, n l^{\prime}\right)}{Z}\right)
$$

With $\sigma\left(N l, n l^{\prime}\right)$ the screen constant relating to these states.

\subsection{Determining the Screen Constant}

To determine the screen constant, we start from the relation:

$$
E(\alpha)=\langle H\rangle(\alpha)=\frac{\langle\Psi(\alpha)|H| \Psi(\alpha)\rangle}{\langle\Psi(\alpha) \mid \Psi(\alpha)\rangle}
$$

And Hamiltonian of the helium isoelectronic series in given by (in atomic units):

$$
H=-\frac{1}{2} \Delta_{1}-\frac{1}{2} \Delta_{2}-\frac{Z}{r_{1}}-\frac{Z}{r_{2}}+\frac{1}{r_{12}}
$$

The average value of this expression (17), while using the closure relation reflecting the fact that the $\left|r_{1}, r_{2}\right\rangle$ kets are continuous bases in the state space of the two electrons:

$$
\iint \mathrm{d} r_{1}^{3} \mathrm{~d} r_{2}^{3}\left|r_{1}, r_{2}\right\rangle\left\langle r_{1}, r_{2}\right|=\mathbb{1}
$$

From this relation we can from (21):

$$
\begin{aligned}
& E(\alpha) \iint \mathrm{d}^{3} r_{1} \mathrm{~d}^{3} r_{2}\left\langle\Psi(\alpha) \| r_{1}, r_{2}\right\rangle \times\left\langle r_{1}, r_{2} \| \Psi(\alpha)\right\rangle \\
& =\iint \mathrm{d}^{3} r_{1} \mathrm{~d}^{3} r_{2}\left\langle\Psi(\alpha) \| r_{1}, r_{2}\right\rangle \hat{H}\left\langle r_{1}, r_{2} \| \Psi(\alpha)\right\rangle
\end{aligned}
$$


The development of (19) gives:

$$
\begin{aligned}
& E(\alpha) \iint \mathrm{d}^{3} r_{1} \mathrm{~d}^{3} r_{2} \Psi\left(r_{1}, r_{2}, \alpha\right) \times \Psi^{*}\left(r_{1}, r_{2}, \alpha\right) \\
& =\iint \mathrm{d}^{3} r_{1} \mathrm{~d}^{3} r_{2} \Psi\left(r_{1}, r_{2}, \alpha\right) \hat{H} \Psi^{*}\left(r_{1}, r_{2}, \alpha\right)
\end{aligned}
$$

The normalization constant denoted $N$ is given by:

$$
N E(\alpha)=\iint \mathrm{d}^{3} r_{1} \mathrm{~d}^{3} r_{2} \Psi\left(r_{1}, r_{2}, \alpha\right) \hat{H} \Psi^{*}\left(r_{1}, r_{2}, \alpha\right)
$$

And from this relation (24), we obtain:

$$
N=\iint \mathrm{d}^{3} r_{1} \mathrm{~d}^{3} r_{2}\left|\left(r_{1}, r_{2}, \alpha\right)\right|^{2}
$$

To facilitate the development of these expressions, we made a change of variable of some parameters of the Equation (20). It was later that we posed in elliptical coordinates:

$$
s=\left(r_{1}+r_{2}\right) ; t=\left(r_{1}-r_{2}\right) ; u=r_{12}
$$

And the element of elementary volume gives:

$$
\mathrm{d} \tau=\mathrm{d} r_{1}^{3} \mathrm{~d} r_{2}^{3}=2 \pi^{2}\left(s^{2}-t^{2}\right) u \mathrm{~d} s \mathrm{~d} t \mathrm{~d} u
$$

Applying these changes of variables in Equation (23) the preceding expression of the normalization constant denoted $N$ is in elliptic coordinate:

$$
\begin{aligned}
N E(\alpha) & =\int_{0}^{\infty} \mathrm{d} s \int_{0}^{s} \mathrm{~d} u \int_{0}^{u} \mathrm{~d} t\left\{u\left(s^{2}-t^{2}\right) \times\left[\left(\frac{\partial \Psi}{\partial s}\right)^{2}+\left(\frac{\partial \Psi}{\partial t}\right)^{2}+\left(\frac{\partial \Psi}{\partial u}\right)^{2}\right]+2\left(\frac{\partial \Psi}{\partial u}\right)\right. \\
& \left.\times\left[s\left(u^{2}-t^{2}\right) \frac{\partial \Psi}{\partial s}+t\left(s^{2}-u^{2}\right) \frac{\partial \Psi}{\partial t}\right]-\Psi^{2}\left[4 Z s u-s^{2}+t^{2}\right]\right\}
\end{aligned}
$$

Since we did not take into account the Coulomb repulsion, so: $\frac{\partial \Psi}{\partial u}=0$.

The normalization constant becomes:

$$
N E(\alpha)=\int_{0}^{\infty} \mathrm{d} s \int_{0}^{s} \mathrm{~d} u \int_{0}^{u} \mathrm{~d} t\left\{u\left(s^{2}-t^{2}\right) \times\left[\left(\frac{\partial \Psi}{\partial s}\right)^{2}+\left(\frac{\partial \Psi}{\partial t}\right)^{2}\right]-\Psi^{2}\left[4 Z s u-s^{2}+t^{2}\right]\right\}
$$

To determine the values of the screen constant $\sigma$ and the variational parameter $\alpha$, we start from this equation, which is the sum of three integral data as follows:

$$
N E(\alpha)=E_{1}(\alpha)+E_{2}(\alpha)+E_{3}(\alpha)
$$

The development of this expression (27) makes it possible to obtain the value of $\sigma$ and $\alpha$ by the formula:

$$
\frac{\mathrm{d} E\left(\alpha_{i}\right)}{\mathrm{d} \alpha_{i}}=0
$$

The expressions corresponding to $E_{1}(\alpha), E_{2}(\alpha)$, and $E_{3}(\alpha)$, are:

$$
E_{1}(\alpha)=\int_{0}^{\infty} \mathrm{d} s \int_{0}^{s} \mathrm{~d} u \int_{0}^{u} \mathrm{~d} t u\left(s^{2}-t^{2}\right) \times\left(\frac{\partial \Psi}{\partial s}\right)^{2}
$$




$$
\begin{aligned}
& E_{2}(\alpha)=\int_{0}^{\infty} \mathrm{d} s \int_{0}^{s} \mathrm{~d} u \int_{0}^{u} \mathrm{~d} t u\left(s^{2}-t^{2}\right) \times\left(\frac{\partial \Psi}{\partial t}\right)^{2} \\
& E_{3}(\alpha)=-\int_{0}^{\infty} \mathrm{d} s \int_{0}^{s} \mathrm{~d} u \int_{0}^{u} \mathrm{~d} t\left[4 Z s u-s^{2}+t^{2}\right] \Psi^{2}
\end{aligned}
$$

The normalization constant is as follows:

$$
N=\int_{0}^{\infty} \mathrm{d} s \int_{0}^{s} \mathrm{~d} u \int_{0}^{u} \mathrm{~d} t u\left(s^{2}-t^{2}\right) \times \Psi^{2}
$$

With these changes of variables, the correlated wave functions of the states $2 \mathrm{~s} n \mathrm{p}, 3 \mathrm{~s} n \mathrm{p}$, and $4 \mathrm{~s} n \mathrm{p}$ become:

$$
\begin{aligned}
& \Psi(2 \mathrm{~s} 2 \mathrm{p})=-\frac{1}{8} \times((s-t) \times(s \times z+t \times z-4)) \times \mathrm{e}^{-\alpha s} \\
& \Psi(3 \mathrm{~s} 3 \mathrm{p})= \frac{1}{1296} \times\left[z \times(s-t) \times(s \times z-t \times z-12) \times\left(s^{2} \times z^{2}+2 \times s \times t \times z^{2}\right.\right. \\
&\left.\left.-18 \times s \times z+t^{2} \times z^{2}-18 \times t \times z+54\right)\right] \times \mathrm{e}^{-\alpha s} \\
& \Psi(4 \mathrm{~s} 4 \mathrm{p})=- \frac{1}{983040} \times\left(z \times(s-t) \times\left(s^{2} \times z^{2}-2 \times s \times t \times z^{2}-40 \times s \times z\right.\right. \\
&+\left.t^{2} \times z^{2}+40 \times t \times z+320\right) \times\left(s^{3} \times z^{3}+3 \times s^{2} \times t \times z^{3}-48 \times s^{2} \times z^{2}\right. \\
&+ 3 \times s \times t^{2} \times z^{3}-96 \times s \times t \times z^{2}+576 \times s \times z+t^{3} \times z^{3} \\
&-\left.\left.48 \times t^{2} \times z^{2}+576 \times t \times z-1536\right)\right) \times \mathrm{e}^{-\alpha s}
\end{aligned}
$$

\section{Results and Discussions}

In this part, the procedure consists of determining the final expressions of energies, the value of the variational parameter $\alpha$, and the screen constant $\sigma$. Since the calculations used are very complex, and require a lot of changes of variables, with matrices to be manipulated, we have found it necessary to make a first call to a computer program with the software matlab. In this program, we first defined the parameter $s, t, u, \alpha$, and $z$ of Equation (23), the expression of the derivative as a function of each parameter, and the square of its derivatives. In a second step, the expressions of $\left(E_{1}, E_{2}, E_{3}\right.$ and $\left.N\right)$ of the Equations (29)-(32), as well as their factorials were defined and detailed expression by expression. Then, to simplify some parameters, a matrix calculation was carried out in this program, and relations between these matrices were made to obtain a simple expression of the Equation (27) in order to apply the formula of the Equation (28) to have the approximate values of the screen constant $\sigma$ and the variational parameter $\alpha$.

\subsection{Expression of the Total Energies}

In the case of the variational calculation of the Modified Atomic Orbital theory (MAOT, the expression of the total energy of the doubly-excited states ( Nsnp) of an orbital is given by the formula (in Rydberg): 


$$
E(N \mathrm{~s} n \mathrm{p})=-\left(\frac{(Z-\sigma(n \mathrm{~s}))^{2}}{N^{2}}\right)-\left(\frac{(Z-\sigma(n \mathrm{p}))^{2}}{n^{2}}\right)
$$

With $N \neq n$ and $\sigma(n \mathrm{~s})=\sigma(n \mathrm{p})$.

In some cases, a corrective factor may be added to this expression to obtain results that are closer to those found in the literature consulted.

Thus the expressions of the states $2 \mathrm{~s} n \mathrm{p}, 3 \mathrm{~s} n \mathrm{p}$, and $4 \mathrm{~s} n \mathrm{p}$ are detailed as follows:

- For the state 2snp:

$$
E(2 \mathrm{~s} 2 \mathrm{p})=-\left(\frac{(Z-\sigma(n \mathrm{~s}))^{2}}{N^{2}}\right)-\left(\frac{(Z-\sigma(n \mathrm{p}))^{2}}{n^{2}}\right)
$$

With $\sigma(n s)=\sigma(n p)$ and $N=n$.

- For the state $3 \mathrm{~s} n \mathrm{p}$ :

$$
E(3 \mathrm{~s} 3 \mathrm{p})=-\left(\frac{(Z-\sigma(n \mathrm{~s}))^{2}}{N^{2}}\right)-\left(\frac{(Z-\sigma(n \mathrm{p}))^{2}}{n^{2}}\right)
$$

With $\sigma(n \mathrm{~s})=\sigma(n \mathrm{p})$ et $N=n$.

- For the state $4 \mathrm{~s} n \mathrm{p}$ :

$$
E(4 \mathrm{~s} 4 \mathrm{p})=-\left(\frac{(Z-\sigma(n \mathrm{~s}))^{2}}{N^{2}}\right)-\left(\frac{(Z-\sigma(n \mathrm{p}))^{2}}{n^{2}}\right)
$$

With $\sigma(n \mathrm{~s})=\sigma(n \mathrm{p})$ and $N=n$.

\subsection{Expression of the Variational Parameter $\alpha$}

The determination of the variational parameter $\alpha$ comes from the expression (28) with:

$$
E\left(\alpha_{i}\right)=\sum_{i=1}^{3}\left(\frac{E_{i}}{N}\right)
$$

Thus the calculation program is presented in the Appendix, and the variational parameter $\alpha$ of the states $2 \mathrm{~s} 2 \mathrm{p}, 3 \mathrm{~s} 3 \mathrm{p}$ and $4 \mathrm{~s} 4 \mathrm{p}$ is given as follows:

$$
\alpha=\frac{n+l+l^{\prime}}{n} Z\left(1-\frac{\sigma\left(N l, n l^{\prime}\right)}{Z}\right)
$$

For the state 2s2p:

$$
\alpha_{2}(2 \mathrm{~s} 2 \mathrm{p}) \approx \frac{3}{2} Z\left(1-\frac{1}{2} \times \frac{1}{Z}\right)
$$

With $\sigma(2 \mathrm{~s} 2 \mathrm{p})=0.5$.

For the state $3 \mathrm{~s} 3 \mathrm{p}$ :

$$
\alpha_{3}(3 \mathrm{~s} 3 p) \approx \frac{4}{3} Z\left(1-\frac{3}{12} \times \frac{1}{Z}\right)
$$

With $\sigma(3 \mathrm{~s} 3 p)=0.25$. 
For the state $4 \mathrm{~s} 4 \mathrm{p}$ :

$$
\alpha_{4}(4 s 4 p) \approx \frac{5}{4} Z\left(1-\frac{3}{7} \times \frac{1}{Z}\right)
$$

With $\sigma(4 s 4 p)=0.428$.

\subsection{Results and Discussion}

In this work, the results obtained are compared with those found in the theoretical and experimental literature. We have calculated the total energies of the states $\left(3 s n p^{1} \mathrm{P}^{o}\right),\left(2 s n p^{1} \mathrm{P}^{o}\right),\left(4 \mathrm{~s} n \mathrm{p}^{1} \mathrm{P}^{\mathrm{o}}\right)$ as well as the excitation energies of the states $\left(3 s n p{ }^{1} \mathrm{P}^{\mathrm{o}}\right),\left(2 \mathrm{~s} n \mathrm{p}{ }^{1} \mathrm{P}^{\mathrm{o}}\right),\left(4 \mathrm{~s} n \mathrm{p}{ }^{1} \mathrm{P}^{\mathrm{o}}\right)$. For the states $\left(3 \mathrm{snp}{ }^{1} \mathrm{P}^{\mathrm{o}}\right)$ the total energies are given in Rydberg and in $\mathrm{eV}$, shown in Table 1 ( $1 \mathrm{eV}=13.605698$ Ryd) shown in Table 2 . For the $\left(2 \mathrm{~s} n \mathrm{p}{ }^{1} \mathrm{P}^{\mathrm{o}}\right),\left(4 \mathrm{~s} n \mathrm{p}{ }^{1} \mathrm{P}^{\mathrm{o}}\right)$ states, their results are given in $\mathrm{eV}$ and represented in Table 3 and Table 4 respectively. Equations (37), (38), (39) have been used for the calculation of its total states energies $\left(3 \mathrm{~s} n \mathrm{p}{ }^{1} \mathrm{P}^{\mathrm{o}}\right)$, $\left(2 \mathrm{~s} n \mathrm{p}{ }^{1} \mathrm{P}^{\mathrm{o}}\right),\left(4 \mathrm{~s} n \mathrm{p}{ }^{1} \mathrm{P}^{\mathrm{o}}\right)$ respectively. About excitations energies, we have taken the energies of the ground state given by Frankowski and Pekeris [29]. These energies are given in ua ( 1 ua $=2$ Rydberg) their values are given as follows: He (-2.90372), $\mathrm{Li}^{+}$(-7.27991), $\mathrm{Be}^{2+}$ (-13.65556), $\mathrm{Be}^{3+}$ (-22.03097).

In Tables 1-4, we used the variational computation of the modified atomic orbitals theory (MAOT) of the energies doubly-excited states $\left(3 s n p{ }^{1} \mathrm{P}^{\circ}\right),\left(2 s n p{ }^{1} \mathrm{P}^{o}\right)$, (4snp ${ }^{1} \mathrm{P}^{\mathrm{o}}$ ). We compared the results obtained with theoretical results for all of these states, and experimental results existing only for the $\left(3 \mathrm{~s} 3 \mathrm{p}^{1} \mathrm{P}^{o}\right),\left(2 \mathrm{~s} 2 \mathrm{p}{ }^{1} \mathrm{P}^{o}\right)$, helium (He) states of Kossmann et al. [17], (2s $\left.2 \mathrm{p}^{1} \mathrm{P}^{\circ}\right)$, lithium $\left(\mathrm{Li}^{+}\right)$from Diehl et al. [19], and $\left(4 \mathrm{~s} 4 \mathrm{p}^{1} \mathrm{P}^{\circ}\right)$ from Woodru et al. [30]. The theoretical results to which we have compared our results are those of Sakho et al. [25], Ivanov and Safronova [15], Drake and Dalgarno [22], Ho [18], Biaye et al. [21], Bachau

Table 1 . The total energies of the doubly excited states of $(N s n p){ }^{1} \mathrm{P}^{o}$ helium-like systems $(Z=2$ to 10$)$ in Rydberg $(1 \mathrm{Ryd}=13.60569 \mathrm{eV})$.

\begin{tabular}{|c|c|c|c|c|c|c|c|c|c|c|}
\hline \multirow{2}{*}{ States } & & \multicolumn{9}{|c|}{$\mathrm{Z}$} \\
\hline & & 2 & 3 & 4 & 5 & 6 & 7 & 8 & 9 & 10 \\
\hline \multirow{4}{*}{$3 s 3 p^{1} \mathrm{P}^{o}$} & $-E^{p}$ & 0.68056 & 1.68056 & 3.12500 & 5.01389 & 7.34722 & 10.12500 & 13.34722 & 17.01389 & 21.12500 \\
\hline & $-E^{s}$ & 0.66054 & 1.64784 & 3.07958 & 4.95577 & 7.27640 & 10.04147 & 13.25099 & 16.90496 & 21.00337 \\
\hline & $-\mathrm{E}^{\mathrm{a}}$ & 0.67140 & 1.65940 & 3.09000 & 4.96600 & 7.28600 & 10.04800 & 13.25600 & 16.91000 & 21.00000 \\
\hline & $-E^{b}$ & 0.66268 & 1.67395 & 3.15417 & 5.10468 & 7.52607 & 10.41871 & 13.78253 & 17.61787 & 21.92494 \\
\hline \multirow{3}{*}{$3 \mathrm{~s} 4 \mathrm{p}^{1} \mathrm{P}^{\mathrm{o}}$} & $-E^{p}$ & 0.53168 & 1.31293 & 2.44141 & 3.91710 & 5.74002 & 7.91016 & 10.42752 & 13.29210 & 16.50391 \\
\hline & $-E^{s}$ & 0.53206 & 1.31269 & 2.44053 & 3.91561 & 5.73790 & 7.90742 & 10.42415 & 13.28811 & 16.49930 \\
\hline & $-E^{a}$ & 0.54240 & 1.31960 & 2.44400 & 3.91400 & 5.73000 & 7.89600 & 10.40800 & 13.26600 & 16.47200 \\
\hline \multirow{2}{*}{$3 s 5 p^{1} P^{o}$} & $-E^{p}$ & 0.46278 & 1.14278 & 2.12500 & 3.40944 & 4.99611 & 6.88500 & 9.07611 & 11.56944 & 14.36500 \\
\hline & $-E^{s}$ & 0.47259 & 1.15756 & 2.14475 & 3.43416 & 5.02579 & 6.91965 & 9.11573 & 11.61403 & 14.41455 \\
\hline
\end{tabular}

P: Present results obtained from Equation (38); s: (Sakho et al., 2010) [26], a: (Bachau et al., 1991) [20]; b: (Biaye et al., 2005) [21]. 
Table 2. The total energies of the doubly excited states of $(N s n \mathrm{p})^{1} \mathrm{P}^{\mathrm{o}}$ types of helium-like systems $(Z=2$ to 10$)$. Results given in $\mathrm{eV}(1 \mathrm{Ryd}=13.60569 \mathrm{eV})$.

\begin{tabular}{|c|c|c|c|c|c|c|c|c|c|c|}
\hline \multirow{2}{*}{ States } & \multicolumn{10}{|c|}{$Z$} \\
\hline & & 2 & 3 & 4 & 5 & 6 & 7 & 8 & 9 & 10 \\
\hline \multirow{6}{*}{$3 s 3 p^{1} \mathrm{P}^{o}$} & $-E^{p}$ & 9.26 & 22.87 & 42.52 & 68.22 & 99.96 & 137.76 & 181.60 & 231.49 & 287.42 \\
\hline & $-\mathrm{E}^{\mathrm{s}}$ & 9.10 & 22.47 & 41.88 & 67.34 & 98.85 & 136.40 & 180.01 & 229.66 & 285.35 \\
\hline & $-\mathrm{E}^{\mathrm{h}}$ & 8.28 & 21.14 & 40.05 & 65.01 & 96.01 & 133.06 & 176.16 & 225.30 & 280.49 \\
\hline & $-\mathrm{E}^{\mathrm{k}}$ & 9.10 & 22.33 & 42.04 & 67.52 & 99.03 & & & & \\
\hline & $-\mathrm{E}^{1}$ & 9.11 & 22.54 & 42.00 & 67.51 & & & & & \\
\hline & $-\mathrm{E}^{\mathrm{i}}$ & 9.10 & & & & & & & & \\
\hline \multirow{2}{*}{$3 s 4 p^{1} \mathrm{P}^{o}$} & $-E^{p}$ & 7.23 & 17.86 & 33.22 & 53.29 & 78.10 & 107.62 & 141.87 & 180.85 & 224.55 \\
\hline & $-\mathrm{E}^{\mathrm{s}}$ & 7.66 & 18.42 & 33.90 & 54.10 & 79.03 & 108.68 & 143.06 & 182.16 & 225.98 \\
\hline \multirow{2}{*}{$3 \mathrm{~s} 5 \mathrm{p}^{1} \mathrm{P}^{\mathrm{o}}$} & $-\mathrm{E}^{\mathrm{p}}$ & 6.30 & 15.55 & 28.91 & 46.39 & 67.98 & 93.68 & 123.49 & 157.41 & 195.45 \\
\hline & $-\mathrm{E}^{\mathrm{s}}$ & 7.02 & 16.58 & 30.25 & 48.03 & 69.93 & 95.94 & 126.05 & 160.05 & 198.63 \\
\hline
\end{tabular}

P: Present results obtained from Equation (38); s: (Sakho et al., 2008) [25]; h: (Ivanov and Safronova, 1993) [16]; i: experimental results (Kossmann et al., 1988) [17]; k: (Wagué, 1987) [31]; 1: (Lipsky et al., 1977) [32].

Table 3. The total energies of the doubly excited states of (Ns np) ${ }^{1} \mathrm{P}^{\mathrm{o}}$ types of helium-like systems $(Z=2$ to 10$)$. Results given in $\mathrm{eV}(1 \mathrm{Ryd}=13.60569 \mathrm{eV})$.

\begin{tabular}{|c|c|c|c|c|c|c|c|c|c|c|}
\hline \multirow{2}{*}{ States } & & \multicolumn{9}{|c|}{$Z$} \\
\hline & & 2 & 3 & 4 & 5 & 6 & 7 & 8 & 9 & 10 \\
\hline \multirow{6}{*}{$2 \mathrm{~s} 2 \mathrm{p}^{1} \mathrm{P}^{o}$} & $-\mathrm{E}^{\mathrm{p}}$ & 17.96 & 46.88 & 89.39 & 145.52 & 215.25 & 298.58 & 395.52 & 506.07 & 630.22 \\
\hline & $-\mathrm{E}^{\mathrm{s}}$ & 18.88 & 47.76 & 90.24 & 146.34 & 216.03 & 299.33 & 396.24 & 506.76 & 630.88 \\
\hline & $\mathrm{E}^{\alpha}$ & 18.86 & 47.82 & 90.33 & 146.40 & 216.07 & 299.32 & 396.18 & 506.64 & 630.70 \\
\hline & $-E^{h}$ & 19.42 & 48.23 & 90.63 & 146.66 & 216.28 & 299.51 & 396.34 & 506.78 & 630.84 \\
\hline & $-E^{j}$ & 18.87 & 47.84 & 90.34 & 146.42 & 216.09 & 299.34 & 396.20 & 506.20 & 630.84 \\
\hline & $-\mathrm{E}^{\mathrm{f,i}}$ & $18.88^{\mathrm{i}}$ & $47.78 f$ & & & & & & & \\
\hline \multirow{3}{*}{$2 \mathrm{~s} 3 \mathrm{p}^{1} \mathrm{P}^{o}$} & $-\mathrm{E}^{\mathrm{p}}$ & 15.05 & 37.16 & 69.09 & 110.85 & 162.44 & 223.86 & 295.10 & 376.16 & 467.06 \\
\hline & $-E^{s}$ & 15.95 & 38.23 & 70.34 & 112.28 & 164.04 & 225.63 & 297.05 & 378.29 & 469.36 \\
\hline & $-\mathrm{E}^{\mathrm{h}}$ & 15.95 & 37.99 & 69.86 & 111.55 & 163.07 & 224.41 & 295.59 & 376.58 & 467.41 \\
\hline
\end{tabular}

P: Present results obtained from Equation (37); s: (Sakho et al., 2008) [25]; a: (Ho, 1980) [18]; h: (Ivanov and Safronova, 1993) [16]; i: experimental results (Kossmann et al., 1988) [17]; f: Experimental data, (Diehl et al., 1999) [19]; j: (Drake and Dalgarno, 1971) [22].

et al. [20], Sakho et al. [26], Wagué [31], Lipsky et al. [32].

Thus in Table 1, Table 2, containing the states $\left(3 \mathrm{snp}{ }^{1} \mathrm{P}^{\circ}\right)$, we have calculated the total energies of doubly-excited states types $\left(3 \mathrm{~s} n \mathrm{p}^{1} \mathrm{P}^{\circ}\right)$ ranging from $(Z=2$ to 10) using Equation (38).

The results found are in perfect agreement with those found in the theoretical and experimental literature consulted and quoted above. For the $\left(3 \mathrm{~s} 3 \mathrm{p}^{1} \mathrm{P}^{\circ}\right)$ helium $(\mathrm{He})$ states, we compared our results with those obtained experimentally by 
Kossman et al. [17], and the results obtained are in perfect agreement.

In Table 3 and Table 4 , containing the states $\left(2 \mathrm{snp}{ }^{1} \mathrm{P}^{\mathrm{o}}\right)$, and $\left(4 \mathrm{~s} 4 \mathrm{p}^{1} \mathrm{P}^{\mathrm{o}}\right)$, we used the Equations ((37), (39)) respectively. Then we added to each of these equations a corrective factor to obtain results equivalent to those found in the theoretical and experimental. In Table 3, states $\left(2 \operatorname{snp}{ }^{1} \mathrm{P}^{\circ}\right)$, we calculated the total energies of doubly excited states of helium-like systems $(Z=2$ to 10$)$. Our results found are in good agreement with the theoretical results [16] [22] [25] and experimental [17] [19].

In Table 4 , states $\left(4 \mathrm{~s} 4 \mathrm{p}{ }^{1} \mathrm{P}^{\circ}\right)$, we also calculated the total energies of doubly-excited states of helium-like systems $(Z=2$ to 10$)$. The results found are in

Table 4. The total energies of the doubly excited states of (Ns np $)^{1} \mathrm{P}^{\mathrm{o}}$ types of helium-like systems $(Z=2$ to 10$)$. Results given in $\mathrm{eV}(1 \mathrm{Ryd}=13.60569 \mathrm{eV})$.

\begin{tabular}{|c|c|c|c|c|c|c|c|c|c|c|}
\hline \multirow{2}{*}{ State } & \multicolumn{10}{|c|}{$\mathrm{Z}$} \\
\hline & & 2 & 3 & 4 & 5 & 6 & 7 & 8 & 9 & 10 \\
\hline \multirow{4}{*}{$4 s 4 p^{1} \mathrm{P}^{o}$} & $-E^{p}$ & 5.24 & 12.91 & 23.98 & 38.46 & 56.33 & 77.61 & 102.29 & 130.37 & 161.85 \\
\hline & $-\mathrm{E}^{\mathrm{s}}$ & 5.35 & 13.03 & 24.10 & 38.58 & 56.46 & 77.75 & 102.43 & 132.03 & 162.00 \\
\hline & $\mathrm{E}^{\alpha}$ & 5.29 & 12.95 & 24.01 & 38.46 & 56.31 & 77.56 & 102.43 & 130.27 & 161.72 \\
\hline & $-\mathrm{E}^{\mathrm{m}}$ & 5.35 & & & & & & & & \\
\hline
\end{tabular}

P: Present results obtained from Equation (39); m: Experimental data, Woodruff and Samson (1982) [30].

Table 5. Excitation energies of the doubly excited states of (Nsnp) ${ }^{1} \mathrm{P}^{o}$ types of helium-like systems $(Z=2$ to 5$)$. Results given in eV (1 Ryd = $13.60569 \mathrm{eV} ; 1$ ua $=2$ Rydberg).

\begin{tabular}{|c|c|c|c|c|c|}
\hline \multirow{2}{*}{ States } & & \multicolumn{4}{|c|}{$Z$} \\
\hline & & 2 & 3 & 4 & 5 \\
\hline \multirow{4}{*}{$2 \mathrm{~s} 2 \mathrm{p}^{1} \mathrm{P}^{\mathrm{o}}$} & $\mathrm{E}^{\mathrm{p}}$ & 61.05 & 151.22 & 282.19 & 453.98 \\
\hline & $E^{s}$ & 60.13 & 150.34 & 281.35 & 453.15 \\
\hline & $\mathrm{E}^{\mathrm{j}}$ & 60.13 & & & \\
\hline & $E^{f, i}$ & $60.13^{\mathrm{i}}$ & $150.31^{\mathrm{f}}$ & & \\
\hline \multirow{2}{*}{$2 \mathrm{~s} 3 \mathrm{p}^{1} \mathrm{P}^{o}$} & $E^{p}$ & 63.97 & 160.94 & 302.50 & 488.64 \\
\hline & $\mathrm{E}^{\mathrm{s}}$ & 63.06 & 159.87 & 301.25 & 487.21 \\
\hline \multirow{3}{*}{$3 \mathrm{~s} 3 \mathrm{p}^{1} \mathrm{P}^{\mathrm{o}}$} & $\mathrm{E}^{\mathrm{p}}$ & 69.75 & 175.23 & 329.07 & 531.28 \\
\hline & $\mathrm{E}^{\mathrm{s}}$ & 69.91 & 175.63 & 330.54 & 532.15 \\
\hline & $\mathrm{E}^{\mathrm{i}}$ & 69.91 & & & \\
\hline \multirow{2}{*}{$3 s 4 p^{1} P^{o}$} & $E^{p}$ & 71.78 & 180.23 & 338.37 & 546.20 \\
\hline & $\mathrm{E}^{\mathrm{s}}$ & 71.35 & 179.68 & 337.69 & 545.39 \\
\hline \multirow{2}{*}{$3 s 5 p^{1} \mathrm{P}^{o}$} & $\mathrm{E}^{\mathrm{p}}$ & 72.72 & 182.55 & 342.67 & 553.11 \\
\hline & $\mathrm{E}^{\mathrm{s}}$ & 71.99 & 181.52 & 341.34 & 551.46 \\
\hline \multirow{3}{*}{$4 \mathrm{~s} 4 \mathrm{p}^{1} \mathrm{P}^{\mathrm{o}}$} & $E^{p}$ & 73.78 & 185.19 & 347.60 & 561.04 \\
\hline & $\mathrm{E}^{\mathrm{s}}$ & 73.66 & 185.07 & 347.49 & 560.91 \\
\hline & $\mathrm{E}^{\mathrm{m}}$ & 73.66 & & & \\
\hline
\end{tabular}


perfect agreement with those found in the literature consulted.

In Table 5, we presented the excitation energies of the doubly-excited states of $\left(N s n \mathrm{p}{ }^{1} \mathrm{P}^{\circ}\right)(N, n \leq 5)$ types of helium and its assimilated ions. They are calculated from the energies of the ground state given by Frankowski and Pekeris [29]. The results found in this table are in perfect agreement with the results found by the other authors.

\section{Conclusion}

In a global way, we applied the variational procedure of the modified atomic orbitals theory for the computation of total energies and excitation senergies doubly-excited states of the atomic system with several electrons. In order to achieve our results, we used a matlab program for the first time to reduce the complexity of the calculations. This program allowed us to determine the approximate expressions of the variational parameter, and of the screen constant.

\section{Conflicts of Interest}

The authors declare no conflicts of interest regarding the publication of this paper.

\section{References}

[1] Madden, R.P. and Codling, K. (1963) Physical Review Letters, 10, 516. https://doi.org/10.1103/PhysRevLett.10.516

[2] Codling, K., Madden, R.P. and Ederer, D.L. (1967) Physical Review, 155, 26. https://doi.org/10.1103/PhysRev.155.26

[3] Cooper, J.W., Fano, U. and Prats, F. (1963) Physical Review Letters, 10, 518. https://doi.org/10.1103/PhysRevLett.10.518

[4] Kahn, S.M. (1999) Physica Scripta T, 80, 23. https://doi.org/10.1515/labm.1999.23.2.80

[5] Fano, U. (1983) Reports on Progress in Physics, 46, 97. https://doi.org/10.1088/0034-4885/46/2/001

[6] Doschek, A.G., Meeking, F.J., Kreplin, W.R., Chubb, A.T. and Friedman, H. (1971) The Astrophysical Journal, 164, 165. https://doi.org/10.1086/150827

[7] Walker, C.B.A. and Rugge, R.H. (1971) The Astrophysical Journal, 164, 181. https://doi.org/10.1086/150828

[8] Buckman, S.J., Hammond, P., Read, F.H. and King, G.C. (1983) Journal of Physics $B, 16,4039$. https://doi.org/10.1088/0022-3700/16/21/024

[9] Buckman, S.J. and Newman, D.S. (1987) Journal of Physics B, 20, 711. https://doi.org/10.1088/0022-3700/20/21/008

[10] Rau, P.R.A. (1990) Reports on Progress in Physics, 53, 181. https://doi.org/10.1088/0034-4885/53/2/002

[11] Sinanoglu, O. and Herrick, R.D. (1975) The Journal of Chemical Physics, 62, 886. https://doi.org/10.1063/1.430540

[12] Herrick, R.D. (1980) Physical Review A, 22, 1346. https://doi.org/10.1103/PhysRevA.22.1346

[13] Herrick, D.R., Kellman, M.E. and Poliak, R.D. (1980) Physical Review A, 22, 1517. 
https://doi.org/10.1103/PhysRevA.22.1517

[14] Condon, E.U. and Shortley, G.H. (1963) Theory of Atomic Spectra. Cambridge University Press, Cambridge.

[15] Kuhn, H.G. (1969) Atomic Spectra. 2nd Edition, Longman Group Ltd., London.

[16] Ivanov, I.A. and Safronova, U.I. (1993) Optics and Spectroscopy, 75, 298-304.

[17] Kossmann, H., Krassig, B. and Schmidt, V. (1988) Journal of Physics B: Atomic, Molecular and Optical Physics, 21, 1489.

https://doi.org/10.1088/0953-4075/21/9/009

[18] Ho, Y.K. (1980) Physics Letters A, 79, 44-46. https://doi.org/10.1016/0375-9601(80)90313-8

[19] Diehl, S., Cubaynes, D., Bizau, J.M., Wuilleumier, F.J., Kennedy, E.T., Mosnier, J.P., and Morgan, T.J. (1999) Journal of Physics B: Atomic, Molecular and Optical Physics, 32, 4193. https://doi.org/10.1088/0953-4075/32/17/305

[20] Bachau, H., Martin, F., Riera, A. and Yanez, M. (1991) Atomic Data and Nuclear Data Tables, 48, 167-212. https://doi.org/10.1016/0092-640X(91)90006-P

[21] Biaye, M., Konte, A., Ndao, A.S. and Wague, A. (2005) Physica Scripta, 72, 373. https://doi.org/10.1238/Physica.Regular.072a00373

[22] Drake, G.W.F. and Dalgarno, A. (1971) Proceedings of the Royal Society of London. A. Mathematical and Physical Sciences, 320, 549-560. https://doi.org/10.1098/rspa.1971.0009

[23] Wague, A. (1990) Zeitschrift für Physik D Atoms, Molecules and Clusters, 15, 199-202. https://doi.org/10.1007/BF01437180

[24] Ray, D. and Mukherjee, P.K. (1991) Journal of Physics B: Atomic, Molecular and Optical Physics, 24, 1241. https://doi.org/10.1088/0953-4075/24/6/013

[25] Sakho, I., Ndao, A.S., Biaye, M. and Wague, A. (2008) The European Physical Journal $D$, 47, 37-44. https://doi.org/10.1140/epjd/e2008-00018-2

[26] Sakho, I., Konte, A., Ndao, A.S., Biaye, M. and Wagué, A. (2010) Physica Scripta, 82, Article ID: 035301. https://doi.org/10.1088/0031-8949/82/03/035301

[27] Slater, J.C. (1930) Physical Review, 36, 57. https://doi.org/10.1103/PhysRev.36.57

[28] Abdourahmane, D., Ba, M.D., Badiane, J.K., Gning, M.T., Sow, M. and Sakho, I. (2018) Journal of Modern Physics, 9, 2594-2622. https://doi.org/10.4236/jmp.2018.914162

[29] Frankowski, F. and Pekeris, L.C. (1966) Physical Review, 146, 46. https://doi.org/10.1103/PhysRev.146.46

[30] Woodruff, D.R. and Samson, J.A.R. (1982) Physical Review Letters, 25, 848. https://doi.org/10.1103/PhysRevA.25.848

[31] Wagué, A. (1987) Zeitschrift für Physik D Atoms, Molecules and Clusters, 6, 337-344. https://doi.org/10.1007/BF01437060

[32] Lipsky, L., Anania, R. and Conneely, M.J. (1977) Atomic Data and Nuclear Data Tables, 20, 127-141. https://doi.org/10.1016/0092-640X(77)90042-0 


\section{Appendix}

\section{Appendix A: Calculation Procedure for the Determination of the Radial Wave Function}

The procedure for determining the radial wave function is given as follows:

$$
R_{n, l}(r)=\left\{\left(\frac{2 Z}{n a_{0}}\right)^{3} \frac{(n-l-1) !}{2 n[(n+l) !]^{3}}\right\}^{1 / 2} \mathrm{e}^{-\frac{z r}{n a_{0}}}\left(\frac{2 Z r}{n a_{0}}\right)^{l} L_{n+l}^{2 l+1}\left(\frac{2 Z r}{n a_{0}}\right)
$$

The associated Laguerre polynomials are linked to the Laguerre polynomials $L_{n+l}(r)$ by the Rodrigue formula:

$$
\begin{aligned}
& L_{n}^{k}(r)=(-1) \frac{\mathrm{d}^{k}}{\mathrm{~d} r^{k}} L_{n}(r) \\
& L_{n}(r)=\mathrm{e}^{r} \frac{\mathrm{d}^{n}}{\mathrm{~d} r^{n}}\left(r^{n} \mathrm{e}^{-r}\right)
\end{aligned}
$$

For different values of $n$ and $l$, the Laguerre polynomials are mutually orthogonal, which then determines the orthogonality of the radial wave functions.

Let's give the example of the $4 \mathrm{~s}$ wave function:

For the state 4 s we have: $n=4, l=0$

$$
L_{n+l}^{2 l+1}\left(\frac{2 Z r}{n a_{o}}\right)=L_{4}^{1}\left(\frac{2 Z r}{n a_{o}}\right) \Rightarrow \frac{\mathrm{d}}{\mathrm{d} r}\left(L_{4}(r)\right)\left(\frac{2 Z r}{n a_{o}}\right)
$$

And;

$$
L_{4}=\mathrm{e}^{r} \frac{\mathrm{d}^{4}}{\mathrm{~d} r^{4}}\left(r^{n} \mathrm{e}^{-r}\right)
$$

By developing this expression, we get:

$$
\begin{gathered}
L_{4}(r)=\mathrm{e}^{r} \frac{\mathrm{d}^{3}}{\mathrm{~d} r^{3}}\left(4 r^{3} \mathrm{e}^{-r}-r^{4} \mathrm{e}^{-r}\right)=\mathrm{e}^{r} \frac{\mathrm{d}^{2}}{\mathrm{~d} r^{2}}\left(12 r^{2} \mathrm{e}^{-r}-4 r^{3} \mathrm{e}^{-r}-4 r^{3} \mathrm{e}^{-r}+r^{4} \mathrm{e}^{-r}\right) \\
\begin{array}{c}
L_{4}(r)=\mathrm{e}^{r} \frac{\mathrm{d}}{\mathrm{d} r}\left(24 r \mathrm{e}^{-r}-12 r^{2} \mathrm{e}^{-r}-12 r^{2} \mathrm{e}^{-r}+4 r^{3} \mathrm{e}^{-r}\right. \\
\left.-12 r^{2} \mathrm{e}^{-r}+4 r^{3} \mathrm{e}^{-r}+4 r^{3} \mathrm{e}^{-r}-r^{4} \mathrm{e}^{-r}\right)
\end{array} \\
\begin{aligned}
L_{4}(r)=\mathrm{e}^{r}\left(24 \mathrm{e}^{-r}-24 r \mathrm{e}^{-r}-24 r \mathrm{e}^{-r}+12 r^{2} \mathrm{e}^{-r}-24 r \mathrm{e}^{-r}+12 r^{2} \mathrm{e}^{-r}\right. \\
+12 r^{2} \mathrm{e}^{-r}-4 r^{3} \mathrm{e}^{-r}-24 r \mathrm{e}^{-r}+12 r^{2} \mathrm{e}^{-r}+12 r^{2} \mathrm{e}^{-r}-4 r^{3} \mathrm{e}^{-r} \\
\left.+12 r^{2} \mathrm{e}^{-r}-4 r^{3} \mathrm{e}^{-r}-4 r^{3} \mathrm{e}^{-r}+r^{4} \mathrm{e}^{-r}\right) \\
L_{4}(r)=\left(24-96 r+72 r^{2}-16 r^{3}+r^{4}\right)
\end{aligned}
\end{gathered}
$$

Then he comes:

$$
L_{4}^{1}=\frac{\mathrm{d}}{\mathrm{d} r} L_{4}(r)=4\left(-24+36 \times r-12 \times r^{2}+r^{3}\right)
$$

Which give, 


$$
\begin{aligned}
& L_{n+l}^{2 l+1}\left(\frac{2 Z \times r}{n a_{0}}\right)=L_{4}^{1}\left(\frac{2 Z \times r}{n a_{0}}\right) \\
& =(-4) \times\left(-24 \times\left(\frac{2 Z}{4 a_{0}}\right)^{0}+36 \times r\left(\frac{2 Z}{4 a_{0}}\right)^{1}-12 \times r^{2}\left(\frac{2 Z}{4 a_{0}}\right)^{2}+r^{3}\left(\frac{2 Z}{4 a_{0}}\right)^{3}\right)
\end{aligned}
$$

So the determination of the first part of the expression (A1)

$$
\left\{\left(\frac{2 Z}{n a_{0}}\right)^{3} \times \frac{(n-l-1) !}{2 n \times[(n+1) !]^{3}}\right\}^{\frac{1}{2}}
$$

For $n=4$ and $l=0$, we have:

$$
\begin{gathered}
\left\{\left(\frac{2 Z}{n a_{0}}\right)^{3} \frac{(n-l-1) !}{2 n \times((n+l) !)^{3}}\right\}^{\frac{1}{2}}=\left\{\left(\frac{Z}{2 \times a_{0}}\right)^{3} \times\left(\frac{6}{8 \times 24^{3}}\right)\right\}^{\frac{1}{2}} \\
=\left\{\left(\frac{Z}{2 \times a_{0}}\right)^{3} \times\left(\frac{3}{4 \times 24^{3}}\right)^{\frac{1}{2}}\right\}=\left\{\left(\frac{Z}{2 \times a_{0}}\right)^{\frac{3}{2}}\left(\frac{1}{96 \times \sqrt{2}}\right)\right\}=\left\{\frac{1}{4 \times 96}\left(\frac{Z}{a_{0}}\right)^{\frac{3}{2}}\right\} \\
\left\{\left(\frac{2 Z}{n a_{0}}\right)^{3} \frac{(n-l-1) !}{2 n \times((n+l) !)^{3}}\right\}^{\frac{1}{2}}=\left\{\frac{1}{4 \times 96}\left(\frac{Z}{a_{0}}\right)^{\frac{3}{2}}\right\}
\end{gathered}
$$

Thus, starting from (A8) and (A10);

$$
\begin{aligned}
& \left\{\frac{1}{4 \times 96}\left(\frac{Z}{a_{0}}\right)^{\frac{3}{2}}\right\} \times(-4) \times\left(-24+\frac{18 \times Z r}{a_{0}}-\frac{3 \times Z^{2} r^{2}}{a_{0}^{2}}+\frac{Z^{3} r^{3}}{8 a_{0}^{3}}\right) \\
& =\left[\frac{1}{96}\left(24-\frac{18 Z}{a_{0}} r+\frac{32 Z^{2}}{a_{0}^{2}} r^{2}-\frac{Z^{3}}{8 a_{0}^{3}} r^{3}\right)\left(\frac{Z}{a_{0}}\right)^{\frac{3}{2}}\right]
\end{aligned}
$$

Simplifying by 24 we finally obtain the expression of the radial wave function 4s as follows:

$$
R_{4,0}=\frac{24}{96}\left(\frac{Z}{a_{0}}\right)^{\frac{3}{2}}\left(1-\frac{3 Z}{4 a_{0}} r+\frac{Z^{2}}{8 a_{0}^{2}} r^{2}-\frac{Z^{3}}{192 a_{0}^{3}} r^{3}\right) \mathrm{e}^{-\frac{Z \times r}{4 a_{0}}}
$$

By analogy the wave function $4 \mathrm{p}$ is given as follows:

$$
R_{4,1}=\frac{5}{16 \sqrt{2}}\left(\frac{Z}{a_{0}}\right)^{\frac{3}{2}}\left(\frac{Z}{a_{0}} r-\frac{1}{4} \frac{Z^{2}}{a_{0}^{2}} r^{2}+\frac{1}{80} \frac{Z^{3}}{a_{0}^{3}} r^{3}\right) \mathrm{e}^{-\frac{Z \times r}{4 a_{0}}}
$$

\section{Appendix B: Principle of Determining the Screen Constant}

To determine the screen constant, we start from the relation:

$$
E(\alpha)=\langle H\rangle(\alpha)=\frac{\langle\Psi(\alpha)|H| \Psi(\alpha)\rangle}{\langle\Psi(\alpha) \mid \Psi(\alpha)\rangle}
$$


And the Hamiltonian $\mathrm{H}$ (in atomic unit) is:

$$
H=-\frac{1}{2} \Delta_{1}-\frac{1}{2} \Delta_{2}-\frac{z}{r_{1}}-\frac{z}{r_{2}}+\frac{1}{r_{12}}
$$

The average value of this expression (B2), while using the closure relation reflecting the fact that the $\left|r_{1}, r_{2}\right\rangle$ kets are continuous bases in the state space of the two electrons:

$$
\iint \mathrm{d} r_{1}^{3} \mathrm{~d} r_{2}^{3}\left|r_{1}, r_{2}\right\rangle\left\langle r_{1}, r_{2}\right|=11
$$

From this relation we can from (B3):

$$
\begin{aligned}
& E(\alpha) \iint \mathrm{d}^{3} r_{1} \mathrm{~d}^{3} r_{2}\left\langle\Psi(\alpha) \| r_{1}, r_{2}\right\rangle \times\left\langle r_{1}, r_{2} \| \Psi(\alpha)\right\rangle \\
& =\iint \mathrm{d}^{3} r_{1} \mathrm{~d}^{3} r_{2}\left\langle\Psi(\alpha)|| r_{1}, r_{2}\right\rangle \hat{H}\left\langle r_{1}, r_{2} \| \Psi(\alpha)\right\rangle
\end{aligned}
$$

The development of (B4) gives:

$$
\begin{aligned}
& E(\alpha) \iint \mathrm{d}^{3} r_{1} \mathrm{~d}^{3} r_{2} \Psi\left(r_{1}, r_{2}, \alpha\right) \times \Psi^{*}\left(r_{1}, r_{2}, \alpha\right) \\
& =\iint \mathrm{d}^{3} r_{1} \mathrm{~d}^{3} r_{2} \Psi\left(r_{1}, r_{2}, \alpha\right) \hat{H} \Psi^{*}\left(r_{1}, r_{2}, \alpha\right)
\end{aligned}
$$

The normalization constant denoted $N$ is given by:

$$
N E(\alpha)=\iint \mathrm{d}^{3} r_{1} \mathrm{~d}^{3} r_{2} \Psi\left(r_{1}, r_{2}, \alpha\right) \hat{H} \Psi^{*}\left(r_{1}, r_{2}, \alpha\right)
$$

And from this relation (B6), we obtain:

$$
N=\iint \mathrm{d}^{3} r_{1} \mathrm{~d}^{3} r_{2}\left|\left(r_{1}, r_{2}, \alpha\right)\right|^{2}
$$

To facilitate the development of these expressions, we made a change of variable of some parameters of the Equation (B5). It was later that we posed in elliptical coordinates:

$$
s=\left(r_{1}+r_{2}\right) ; t=\left(r_{1}-r_{2}\right) ; u=r_{12}
$$

And the element of elementary volume gives:

We know that, $\mathrm{d} \tau=\mathrm{d} r_{1}^{3} \mathrm{~d} r_{2}^{3}$

$$
\mathrm{d} \tau=\mathrm{d} r_{1}^{3} \mathrm{~d} r_{2}^{3}=2 \pi^{2}\left(s^{2}-t^{2}\right) u \mathrm{~d} s \mathrm{~d} t \mathrm{~d} u
$$

Applying these changes of variables in Equation (B7) the preceding expression of the normalization constant denoted $\mathrm{N}$ is in elliptic coordinate:

$$
\begin{aligned}
N E(\alpha)= & \int_{0}^{\infty} \mathrm{d} s \int_{0}^{s} \mathrm{~d} u \int_{0}^{u} \mathrm{~d} t\left\{u\left(s^{2}-t^{2}\right) \times\left[\left(\frac{\partial \Psi}{\partial s}\right)^{2}+\left(\frac{\partial \Psi}{\partial t}\right)^{2}+\left(\frac{\partial \Psi}{\partial u}\right)^{2}\right]+2\left(\frac{\partial \Psi}{\partial u}\right)\right. \\
& \left.\times\left[s\left(s^{2}-t^{2}\right) \frac{\partial \Psi}{\partial s}+t\left(s^{2}-u^{2}\right) \frac{\partial \Psi}{\partial t}\right]-\Psi^{2}\left[4 Z s u-s^{2}+t^{2}\right]\right\}
\end{aligned}
$$

Since we did not take into account the Coulomb repulsion, so: $\frac{\partial \Psi}{\partial u}=0$

The normalization constant becomes:

$$
N E(\alpha)=\int_{0}^{\infty} \mathrm{d} s \int_{0}^{s} \mathrm{~d} u \int_{0}^{u} \mathrm{~d} t\left\{u\left(s^{2}-t^{2}\right) \times\left[\left(\frac{\partial \Psi}{\partial s}\right)^{2}+\left(\frac{\partial \Psi}{\partial t}\right)^{2}\right]-\Psi^{2}\left[4 Z s u-s^{2}+t^{2}\right]\right\}
$$


To determine the values of the screen constant $\sigma$ and the variational parameter $\alpha$, we start from this equation, which is the sum of three integral data as follows:

$$
N E(\alpha)=E_{1}(\alpha)+E_{2}(\alpha)+E_{3}(\alpha)
$$

The development of this expression (B12) makes it possible to obtain the value of $\sigma$ and $\alpha$ by the formula:

$$
\frac{\mathrm{d} E\left(\alpha_{i}\right)}{\mathrm{d} \alpha_{i}}=0
$$

The expressions corresponding to $E_{1}(\alpha), E_{2}(\alpha)$, and $E_{3}(\alpha)$, are:

$$
\begin{gathered}
E_{1}(\alpha)=\int_{0}^{\infty} \mathrm{d} s \int_{0}^{s} \mathrm{~d} u \int_{0}^{u} \mathrm{~d} t u\left(s^{2}-t^{2}\right) \times\left(\frac{\partial \Psi}{\partial s}\right)^{2} \\
E_{2}(\alpha)=\int_{0}^{\infty} \mathrm{d} s \int_{0}^{s} \mathrm{~d} u \int_{0}^{u} \mathrm{~d} t u\left(s^{2}-t^{2}\right) \times\left(\frac{\partial \Psi}{\partial t}\right)^{2} \\
E_{3}(\alpha)=-\int_{0}^{\infty} \mathrm{d} s \int_{0}^{s} \mathrm{~d} u \int_{0}^{u} \mathrm{~d} t\left[4 Z s u-s^{2}+t^{2}\right] \Psi^{2}
\end{gathered}
$$

The normalization constant is as follows:

$$
N=\int_{0}^{\infty} \mathrm{d} s \int_{0}^{s} \mathrm{~d} u \int_{0}^{u} \mathrm{~d} t u\left(s^{2}-t^{2}\right) \times \Psi^{2}
$$

With these changes of variables, we obtain the equations presented above in section (2.3): Equation $(33 ; 34 ; 35)$. 